\title{
Wie kann man den Klapperstorch anlocken?
}

\author{
Ein unerfüllter Kinderwunsch ist ein Thema, auf das Sie auch in der Hausarztpraxis ange- \\ sprochen werden können. Bevor Sie zum Gynäkologen überweisen, können Sie manchen \\ Paaren schon mit kleinen Tipps zur Lebensgestaltung bzw. Terminplanung weiterhelfen.
}

_ „Bei jedem fünften Paar in Deutschland klappt es nicht mit dem Schwangerwerden“, so Prof. Vanadin Seifert-Klauss vom Klinikum rechts der Isar in München. $\mathrm{Zu}$ den organischen Ursachen, die vom Facharzt abgeklärt werden müssen, gehören eine verminderte Spermienqualität ebenso wie ein fehlender Eisprung. Bei Frauen sollten zudem endokrine Störungen wie ein Diabetes mellitus oder Schilddrüsenfunktionsstörungen ausgeschlossen werden.

\section{Häufig „Terminprobleme“}

„Eine zunehmende Bedeutung bei diesem Problem haben heute aber auch Lebensstilfaktoren“, so SeifertKlauss. Dazu gehören Über- und Untergewicht, Stress, Rauchen, aber auch schlicht „Terminprobleme“, denn Fernbeziehungen sind für die Erfüllung des Kinderwunschs nicht gerade vorteilhaft. Bei Paaren, die zusammenleben, liegt die Wahrscheinlichkeit pro Zyklus, schwanger zu werden, bei $25 \%$. Bei einer Fernbeziehung sinkt die Aussicht auf eine Verwirklichung des Kinderwunsches auf unter 5\%. „Der Eisprung fällt nämlich dummerweise nicht gerade auf das gemeinsame Wochenende, sondern auf einen Tag in der Woche, den man nicht zusammen verbringen kann“, so Seifert-Klauss. So haben viele Paare heute im Gegensatz zu zusammenlebenden Paaren nicht mehr eine Schwangerschaftschance von $75 \%$ in zwölf Monaten.

Für betroffene Paare ist es dann ein Gewinn, wenn man die Ovulation mittels hormoneller Intervention um einige Tage verschieben kann. „Mit Hilfe ein- facher Maßnahmen lässt sich überprüfen, ob und wann eine Ovulation stattgefunden hat“, so Seifert-Klauss. Dann könne man durch eine kurzzeitige Hormontherapie den Eisprung etwas verschieben und dadurch das „Terminproblem“ trotz Fernbeziehung lösen.

\section{Fehlende Ovulation bei PCOS}

12-30\% der jungen Frauen mit normaler Zykluslänge haben keinen oder einen zu seltenen Eisprung. Ein Teil von ihnen leidet an einem Polyzystischen OvarSyndrom (PCOS). Betroffen sind insgesamt 5-8\% der weiblichen Bevölkerung. Bei ihnen reifen im Zyklus 20-30 Follikel heran, die bis zu einem Zentimeter groß werden und im Ultraschall erkennbar sind. Oft kommt es dann aber nicht zu einem Eisprung. Die fehlende Ovulation führt dazu, dass kein Progesteron gebildet wird. Und dieses ist notwendig, um das Endometrium so umzuwandeln, dass sich die befruchtete Eizelle einnisten kann. Begleitsymptome beim PCOS sind Übergewicht, eine vermehrte Körperbehaarung, Akne und Alopezie als Ausdruck einer Hyperandrogenämie.

\section{Hormonelle Intervention}

Was kann man tun, um solchen Frauen mit fehlendem Eisprung zu helfen? „Viele Frauen haben direkt nach dem Absetzen der Pille eine vorübergehende Erhöhung des Follikel-stimulierenden Hormons, und dieser Rebound-Effekt sollte genutzt werden, um schwanger zu werden“, so Seifert-Klauss. Auf ähnliche Weise kann man mit einer zweiwöchi- gen Gestagengabe in der zweiten Zyklushälfte die Wahrscheinlichkeit eines Eisprungs im nächsten Zyklus erhöhen. Das funktioniert aber nur bei Frauen mit einem milden PCOS. Um die so gezielt induzierten Eisprünge für eine Befruchtung nutzen zu können, empfiehlt die Expertin, bereits einige Tage im Vorfeld Geschlechtsverkehr zu haben. „Denn Spermien bleiben im weiblichen Körper drei bis fünf Tage befruchtungsfähig, sodass die Chance besteht, dass sie auf eine Eizelle treffen“, so Seifert-Klauss.

\section{Dr. Peter Stiefelhagen}

- Quelle: 59. Jahrestagung der Deutschen Gesellschaft für Endokrinologie, 26.5.2016 in München

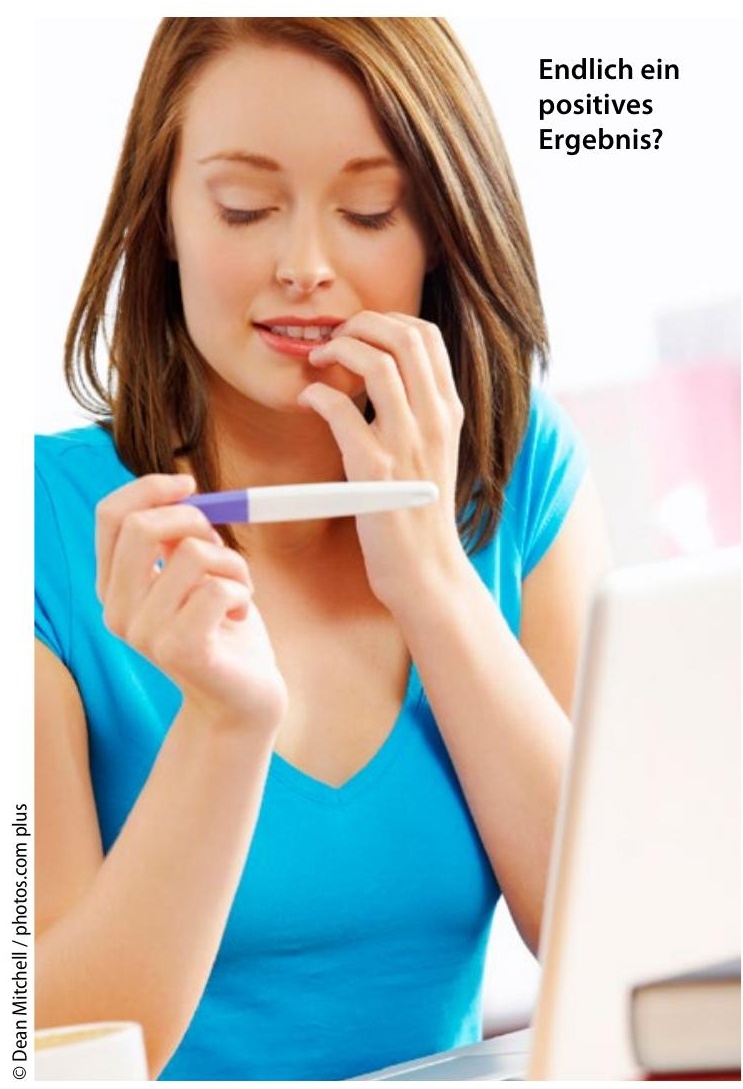

